\title{
On Complete Moment Convergence for Randomly Weighted Sums of NSD Random Variables
}

\author{
Xiang Sun, Zhao-yang Liu, Yan Shen* and Ke-chao Zhang \\ School of Mathematical Science, Anhui University, Hefei 230031, P. R. China.
}

Received March 18, 2018; Accepted December 10, 2018

\begin{abstract}
In this paper, we investigate the complete moment convergence and complete convergence for randomly weighted sums of negatively superadditive dependent (NSD, in short) random variables. The results obtained in the paper generalize the convergence theorem for constant weighted sums to randomly weighted sums of dependent random variables. In addition, strong law of large numbers for NSD sequence is obtained.
\end{abstract}

AMS subject classifications: 60F15.

Key words: Complete moment convergence, randomly weighted, negatively superadditive dependent random variables.

\section{Introduction}

Let $\left\{X_{n}, n \geq 1\right\}$ be a sequence of random variables defined on a fixed probability space $(\Omega, \mathcal{F}, P)$. The concept of negatively superadditive dependent (NSD, in short) random variables was introduced by $\mathrm{Hu}$ [1] based on the class of superadditive functions. Superadditive structure functions have important reliability interpretations, which describe whether a system is more series-like or more parallel-like. The concepts of superadditive structure function and NSD random variables were introduced by Kemperman [2] and $\mathrm{Hu}[1]$ as follows.

Definition 1.1 ([2]). A function $\phi: R^{n} \rightarrow R$ is called superadditive if

$$
\phi(\mathbf{x} \vee \mathbf{y})+\phi(\mathbf{x} \wedge \mathbf{y}) \geq \phi(\mathbf{x})+\phi(\mathbf{y}) \text { for all } \mathbf{x}, \mathbf{y} \in R^{n},
$$

where $\vee$ is for componentwise maximum and $\wedge$ is for componentwise minimum.

*Corresponding author. Email addresses: 206119064@qq.com (X. Sun), 1668892437@qq.com (Z.Y. Liu), shenyan@ahu.edu.cn (Y. Shen), 2055436515@qq. com (K.C. Zhang) 
Definition 1.2 ([1]). A random vector $\mathbf{X}=\left(X_{1}, \ldots, X_{n}\right)$ is said to be negatively superadditive dependent (NSD, in short) if

$$
E \phi\left(X_{1}, \ldots, X_{n}\right) \leq E \phi\left(X_{1}^{*}, \ldots, X_{n}^{*}\right),
$$

where $X_{1}^{*}, \ldots, X_{n}^{*}$ are independent such that $X_{i}^{*}$ and $X_{i}$ have the same distribution for each $i$ and $\phi$ is a superadditive function such that the expectations in (1.1) exist.

Definition 1.3. A sequence of random variables $\left\{X_{n}, n \geq 1\right\}$ is said to be negatively superadditive dependent if for all $n \geq 1,\left(X_{1}, \ldots, X_{n}\right)$ is negatively superadditive dependent.

$\mathrm{Hu}$ [1] gave an example illustrating that NSD random variables are not necessarily negatively associated (NA, in short). Christofides and Vaggelatou [3] indicated that NA random variables are NSD. Negatively superadditive dependent structure is an extension of negatively associated structure and sometimes more useful than negatively associated structure. For example, the structure function of a monotone coherent system can be superadditive [4], so inequalities derived from NSD can give one-side or two-side bounds of the system reliability. The notion of NSD random variables has wide applications in multivariate statistical analysis and reliability theory. Eghbal et al. [5,6] provided some inequalities and strong law of large numbers of quadratic forms of NSD random variables under some assumptions. Shen et al. [7] obtained Khintchine-Kolmogorov-type convergence theorem and strong stability for NSD random variables. Shen et al. [8] discussed the Marcinkiewicz-type strong law of large numbers and integrability of superemum for NSD random variables. Wang et al. [9] and Wang et al. [10] obtained the complete convergence of weighted sums for an array of rowwise NSD random variables. For more details about complete convergence for dependent case, one can refer to Cabrera et al. [11], Yang et al. [12], Li et al. [13] and Wang et al. [14].

The main purpose of this paper is to study the complete moment convergence and complete convergence for randomly weighted sums of NSD random variables. As an application, a strong law of large numbers is obtained for NSD structure.

The following concept of stochastic domination will be used in the main results of the paper.

Definition 1.4 ([15]). A sequence of random variables $\left\{X_{n}, n \geq 1\right\}$ is said to be stochastically dominated by random variable $X$ if there exists a positive constant $C$ such that

$$
P\left(\left|X_{n}\right| \geq x\right) \leq C P(|X| \geq x),
$$

for all $x \geq 0$ and all $n \geq 1$.

Throughout this paper, let $I(A)$ be the indicator of the set $A$ and $X^{+}=\max \{0, X\} . C$ denotes a positive constant which may be different in various places. $a_{n}=O\left(b_{n}\right)$ represents $a_{n} \leq C b_{n}$ for all $n \geq 1$. 


\section{Some lemmas}

The following lemmas will be needed in this paper.

Lemma 2.1 ([1]). If $\left(X_{1}, \ldots, X_{n}\right)$ is NSD and $f_{1}, \ldots, f_{n}$ are all nondecreasing functions, then $\left(f_{1}\left(X_{1}\right), \cdots, f_{n}\left(X_{n}\right)\right)$ is NSD.

Lemma 2.2 ([9]). Let $p>1$ and $\left\{X_{n}, n \geq 1\right\}$ be a sequence of NSD random variables with $E\left|X_{n}\right|^{p}<$ $\infty$ for each $n \geq 1$. Then for all $n \geq 1$,

$$
\begin{array}{ll}
E\left(\max _{1 \leq k \leq n}\left|\sum_{i=1}^{k} X_{i}\right|^{p}\right) \leq 2^{3-p} \sum_{i=1}^{n} E\left|X_{i}\right|^{p}, & \text { for } 1<p \leq 2, \\
E\left(\max _{1 \leq k \leq n}\left|\sum_{i=1}^{k} X_{i}\right|^{p}\right) \leq 2\left(\frac{15 p}{\ln p}\right)^{p}\left[\sum_{i=1}^{n} E\left|X_{i}\right|^{p}+\left(\sum_{i=1}^{n} E X_{i}^{2}\right)^{\frac{p}{2}}\right], & \text { for } p>2 .
\end{array}
$$

Lemma 2.3 ([16]). Let $\left\{Y_{n}, n \geq 1\right\}$ and $\left\{Z_{n}, n \geq 1\right\}$ be sequences of random variables. Then for any $q>1, \varepsilon>0$, and $a>0$,

$$
\begin{aligned}
& E\left(\max _{1 \leq k \leq n}\left|\sum_{i=1}^{k}\left(Y_{i}+Z_{i}\right)\right|-\varepsilon a\right)^{+} \\
\leq & \left(\frac{1}{\varepsilon^{q}}+\frac{1}{q-1}\right) \frac{1}{a^{q-1}}\left(E \max _{1 \leq k \leq n}\left|\sum_{i=1}^{k} Y_{i}\right|^{q}\right)+E\left(\max _{1 \leq k \leq n}\left|\sum_{i=1}^{k} Z_{i}\right|\right) .
\end{aligned}
$$

Lemma 2.4 ([17]). Suppose that $\left\{X_{n}, n \geq 1\right\}$ is a sequence of random variables which is stochastically dominated by a random variable $X$. Then for any $q>0$ and $x>0$,

$$
\begin{aligned}
& E\left|X_{n}\right|{ }^{q} I\left(\left|X_{n}\right| \leq x\right) \leq C\left[E|X|^{q} I(|X| \leq x)+x^{q} P(|X|>x)\right], \\
& E\left|X_{n}\right|^{q} I\left(\left|X_{n}\right|>x\right) \leq C E|X|{ }^{q} I(|X|>x) .
\end{aligned}
$$

\section{Main results}

Theorem 3.1. Assume $\alpha>\frac{1}{2}, p \geq 2$. Let $\left\{X_{n}, n \geq 1\right\}$ be a sequence of NSD random variables which has mean zero and is stochastically dominated by a random variable $X$. Assume that $\left\{A_{n i}, 1 \leq\right.$ $i \leq n, n \geq 1\}$ is an array of independent random variables satisfying that $\left\{A_{n i}, 1 \leq i \leq n, n \geq 1\right\}$ is independent of $\left\{X_{n}, n \geq 1\right\}$. Let $\beta \geq 1$ such that

$$
\sum_{i=1}^{n}\left|A_{n i}\right|^{q}=O\left(n^{\beta}\right)
$$

for some $q>\frac{2(\alpha p-1)}{2 \alpha-1}$. If $E|X|^{p}<\infty$, then for every $\varepsilon>0$,

$$
\sum_{n=1}^{\infty} n^{\alpha p-\alpha-\beta-1} E\left(\max _{1 \leq k \leq n}\left|\sum_{i=1}^{k} A_{n i} X_{i}\right|-\varepsilon n^{\alpha}\right)^{+}<\infty .
$$


Further

$$
\sum_{n=1}^{\infty} n^{\alpha p-\beta-1} P\left(\max _{1 \leq k \leq n}\left|\sum_{i=1}^{k} A_{n i} X_{i}\right|>\varepsilon n^{\alpha}\right)<\infty .
$$

Proof. For $n \geq 1$ and $1 \leq i \leq n$, denote

$$
\begin{aligned}
& X_{n i}=-n^{\alpha} I\left(X_{i}<-n^{\alpha}\right)+X_{i} I\left(\left|X_{i}\right| \leq n^{\alpha}\right)+n^{\alpha} I\left(X_{i}>n^{\alpha}\right), \\
& X_{n i}^{*}=n^{\alpha} I\left(X_{i}<-n^{\alpha}\right)-n^{\alpha} I\left(X_{i}>n^{\alpha}\right)+X_{i} I\left(\left|X_{i}\right|>n^{\alpha}\right),
\end{aligned}
$$

and $\widetilde{X}_{n i}=X_{n i}-E X_{n i}$. Thus $X_{i}=\widetilde{X}_{n i}+E X_{n i}+X_{n i}^{*}$ for $1 \leq i \leq n$.

By Lemma 2.3 with $a=n^{\alpha}$, we obtain that

$$
\begin{aligned}
& \sum_{n=1}^{\infty} n^{\alpha p-\alpha-\beta-1} E\left(\max _{1 \leq k \leq n}\left|\sum_{i=1}^{k} A_{n i} X_{i}\right|-\varepsilon n^{\alpha}\right)^{+} \\
\leq & C \sum_{n=1}^{\infty} n^{\alpha p-\alpha q-\beta-1} E\left(\max _{1 \leq k \leq n}\left|\sum_{i=1}^{k} A_{n i} \widetilde{X}_{n i}\right|^{q}\right)+\sum_{n=1}^{\infty} n^{\alpha p-\alpha-\beta-1} E\left(\max _{1 \leq k \leq n}\left|\sum_{i=1}^{k} A_{n i} X_{n i}^{*}\right|\right) \\
& \quad+\sum_{n=1}^{\infty} n^{\alpha p-\alpha-\beta-1} E\left(\max _{1 \leq k \leq n}\left|\sum_{i=1}^{k} A_{n i} E\left(X_{n i}\right)\right|\right) \\
\doteq & H_{1}+H_{2}+H_{3} .
\end{aligned}
$$

Since $A_{n i} X_{i}=A_{n i}^{+} X_{i}-A_{n i}^{-} X_{i}$, without loss of generality, we can assume that $A_{n i} \geq 0,1 \leq i \leq n$. In view of Lemma 2.1, it is easy to see that $\left\{X_{n i}, 1 \leq i \leq n, n \geq 1\right\}$ is a sequence of NSD random variables. So we have $\left\{A_{n i} \widetilde{X}_{n i}, 1 \leq i \leq n, n \geq 1\right\}$ is a sequence of NSD random variables with mean zero. By $q>\frac{2(\alpha p-1)}{2 \alpha-1}$ and $p \geq 2$, it is easy to see that $q \geq 2$. Thus by Lemma 2.2 we can get that

$$
\begin{aligned}
H_{1} & \leq C \sum_{n=1}^{\infty} n^{\alpha p-\alpha q-\beta-1}\left[\sum_{i=1}^{n} E\left|A_{n i} \widetilde{X}_{n i}\right|^{q}+\left(\sum_{i=1}^{n} E\left(A_{n i} \widetilde{X}_{n i}\right)^{2}\right)^{\frac{q}{2}}\right] \\
& =C \sum_{n=1}^{\infty} n^{\alpha p-\alpha q-\beta-1} \sum_{i=1}^{n} E\left|A_{n i} \widetilde{X}_{n i}\right|^{q}+C \sum_{n=1}^{\infty} n^{\alpha p-\alpha q-\beta-1}\left(\sum_{i=1}^{n} E\left(A_{n i} \widetilde{X}_{n i}\right)^{2}\right)^{\frac{q}{2}} \\
& \doteq H_{11}+H_{12} .
\end{aligned}
$$


By (3.1) and Lemma 2.4, it follows that

$$
\begin{aligned}
H_{11} & \leq C \sum_{n=1}^{\infty} n^{\alpha p-\alpha q-\beta-1} \sum_{i=1}^{n} E\left(\left|A_{n i}\right|^{q}\left|X_{n i}\right|^{q}\right) \\
& =C \sum_{n=1}^{\infty} n^{\alpha p-\alpha q-\beta-1} \sum_{i=1}^{n} E\left|A_{n i}\right|^{q}\left[E\left|X_{i}\right|^{q} I\left(\left|X_{i}\right| \leq n^{\alpha}\right)+n^{\alpha q} E I\left(\left|X_{i}\right|>n^{\alpha}\right)\right] \\
& \leq C \sum_{n=1}^{\infty} n^{\alpha p-\alpha q-1} E|X|^{q} I\left(|X| \leq n^{\alpha}\right)+C \sum_{n=1}^{\infty} n^{\alpha p-1} E I\left(|X|>n^{\alpha}\right) \\
& \doteq H_{11}^{*}+H_{11}^{* *} .
\end{aligned}
$$

Observe that $q>\frac{2(\alpha p-1)}{2 \alpha-1}$ and $p \geq 2$, then we have $q>p$. Therefore it has

$$
\begin{aligned}
H_{11}^{*} & =C \sum_{n=1}^{\infty} n^{\alpha p-\alpha q-1} \sum_{m=1}^{n} E|X|^{q} I\left((m-1)^{\alpha}<|X| \leq m^{\alpha}\right) \\
& =C \sum_{m=1}^{\infty} E|X|^{q} I\left((m-1)^{\alpha}<|X| \leq m^{\alpha}\right) \sum_{n=m}^{\infty} n^{\alpha p-\alpha q-1} \leq C E|X|^{p}<\infty, \\
H_{11}^{* *} & =C \sum_{n=1}^{\infty} n^{\alpha p-1} \sum_{m=n}^{\infty} E I\left(m^{\alpha}<|X| \leq(m+1)^{\alpha}\right) \\
& =C \sum_{m=1}^{\infty} E I\left(m^{\alpha}<|X| \leq(m+1)^{\alpha}\right) \sum_{n=1}^{m} n^{\alpha p-1} \leq C E|X|^{p}<\infty .
\end{aligned}
$$

Combining (3.1) with Hölder's inequality and $q>p \geq 2$, one has

$$
\begin{aligned}
\sum_{i=1}^{n} E A_{n i}^{2} \leq\left(\sum_{i=1}^{n} E\left|A_{n i}\right|^{q}\right)^{\frac{2}{q}}\left(\sum_{i=1}^{n} 1\right)^{1-\frac{2}{q}} \leq n^{\beta \frac{2}{q}+1-\frac{2}{q}}, \\
E X_{n i}^{2}=E\left[\left|X_{i}\right|^{2} I\left(\left|X_{i}\right| \leq n^{\alpha}\right)+n^{2 \alpha} I\left(\left|X_{i}\right|>n^{\alpha}\right)\right] \\
\quad \leq C E\left[X^{2} I\left(|X| \leq n^{\alpha}\right)+X^{2} I\left(|X|>n^{\alpha}\right)\right] \\
\quad=C E X^{2} .
\end{aligned}
$$

Thus, by the fact that $q>\frac{2(\alpha p-1)}{2 \alpha-1}$ it follows that

$$
\begin{aligned}
H_{12} & \leq C \sum_{n=1}^{\infty} n^{\alpha p-\alpha q-\beta-1}\left[\sum_{i=1}^{n}\left(E A_{n i}^{2} E X_{n i}^{2}\right)\right]^{\frac{q}{2}} \\
& \leq C \sum_{n=1}^{\infty} n^{\alpha p-\alpha q-\beta-1} n^{\left(\beta \frac{2}{q}+1-\frac{2}{q}\right) \frac{q}{2}} \\
& \leq C \sum_{n=1}^{\infty} n^{\alpha p-\alpha q+\frac{q}{2}-2}<\infty .
\end{aligned}
$$


For $H_{2}$ and $H_{3}$, similar to the proof of (3.7) and (3.8), we have that

$$
\begin{aligned}
H_{2} & \leq \sum_{n=1}^{\infty} n^{\alpha p-\alpha-\beta-1} \sum_{i=1}^{n} E\left|A_{n i}\right| E\left|X_{n i}^{*}\right| \\
& \leq C \sum_{n=1}^{\infty} n^{\alpha p-\alpha-1} E|X| I\left(|X|>n^{\alpha}\right) \\
& =C \sum_{n=1}^{\infty} n^{\alpha p-\alpha-1} \sum_{m=n}^{\infty} E|X| I\left(m^{\alpha}<|X| \leq(m+1)^{\alpha}\right) \\
& \leq C \sum_{m=1}^{\infty} E\left[|X| I\left(m^{\alpha}<|X| \leq(m+1)^{\alpha}\right)\right] m^{\alpha p-\alpha} \\
& \leq C E|X|^{p}<\infty, \\
H_{3} & \leq \sum_{n=1}^{\infty} n^{\alpha p-\alpha-\beta-1} \sum_{i=1}^{n}\left|E\left(A_{n i} X_{n i}\right)\right| \\
& \leq C \sum_{n=1}^{\infty} n^{\alpha p-\alpha-1} E|X| I\left(|X|>n^{\alpha}\right) \\
& \leq C E|X|^{p}<\infty .
\end{aligned}
$$

Combining (3.4)-(3.11), we can obtain (3.2) immediately. By (3.2) and [16], it follows that

$$
\begin{aligned}
\infty & >\sum_{n=1}^{\infty} n^{\alpha p-\alpha-\beta-1} E\left(\max _{1 \leq k \leq n}\left|\sum_{i=1}^{k} A_{n i} X_{i}\right|-\varepsilon n^{\alpha}\right)^{+} \\
& \geq \sum_{n=1}^{\infty} n^{\alpha p-\alpha-\beta-1} \int_{0}^{\varepsilon n^{\alpha}} P\left(\max _{1 \leq k \leq n}\left|\sum_{i=1}^{k} A_{n i} X_{i}\right|-\varepsilon n^{\alpha}>t\right) d t \\
& \geq \varepsilon \sum_{n=1}^{\infty} n^{\alpha p-\beta-1} P\left(\max _{1 \leq k \leq n}\left|\sum_{i=1}^{k} A_{n i} X_{i}\right|>2 \varepsilon n^{\alpha}\right)
\end{aligned}
$$

which implies (3.3).

If taking $\alpha p=1+\beta, \frac{1}{2}<\alpha<\frac{1+\beta}{2}$, then we get the following Corollary.

Corollary 3.1. Let $\alpha p=1+\beta, \frac{1}{2}<\alpha<\frac{1+\beta}{2}$. Let $\left\{X_{n}, n \geq 1\right\}$ be a sequence of NSD random variables which has mean zero and is stochastically dominated by a random variable $X$. Assume that $\left\{A_{n i}, 1 \leq i \leq n, n \geq 1\right\}$ is an array of independent random variables satisfying that $\left\{A_{n i}, 1 \leq i \leq n, n \geq 1\right\}$ is independent of $\left\{X_{n}, n \geq 1\right\}$. Let $\beta \geq 1$ such that

$$
\sum_{i=1}^{n}\left|A_{n i}\right|^{q}=O\left(n^{\beta}\right)
$$


for some $q>\frac{2 \beta}{2 \alpha-1}$. If $E|X|^{p}<\infty$, then for every $\varepsilon>0$,

$$
\sum_{n=1}^{\infty} n^{-\alpha} E\left(\max _{1 \leq k \leq n}\left|\sum_{i=1}^{k} A_{n i} X_{i}\right|-\varepsilon n^{\alpha}\right)^{+}<\infty .
$$

Further

$$
\sum_{n=1}^{\infty} P\left(\max _{1 \leq k \leq n}\left|\sum_{i=1}^{k} A_{n i} X_{i}\right|>\varepsilon n^{\alpha}\right)<\infty .
$$

By Borel-Cantelli lemma and (3.14), the strong law of large numbers can be obtained as follows.

Corollary 3.2. Under the conditions of Corollary 3.1, we have

$$
\lim _{n \rightarrow \infty} \frac{1}{n^{\alpha}} \max _{1 \leq k \leq n}\left|\sum_{i=1}^{k} A_{n i} X_{i}\right|=0, \quad \text { a.s. }
$$

Remark 3.1. Theorem 3.1 and Corollary 3.1 present complete moment convergence and complete convergence for randomly weighted sums of NSD random variables, which extend the results of Shen et al. ([8]) and Wang et al. ([9]). Note that in [8, 9] the complete moment convergence for constant weighted sums of NSD random variables were obtained.

\section{Acknowledgments}

The authors are most grateful to the editor and the anonymous referees for their careful reading and insightful comments. This work is supported by the National Natural Science Foundation of China under Grant 11671012, 11501005 and 11701004, Natural Science Foundation for Colleges and Universities of Anhui Province under Grant KJ2015A065 and KJ2017A027, Natural Science Foundation of Anhui Province under Grant 1508085J06 and 1608085QA02, Students Science Research Training Program of Anhui University under Grant KYXL2016007, KYXL2017005 and KYXL2017001.

\section{References}

[1] T. Z. Hu, Negatively superadditive dependence of random variables with applications, Chinese J. Appl. Probab. Statist., 16 (2000), 133-144.

[2] J. H. B. Kemperman, On the FKG-inequalities for measures on a partially ordered space, Indag. Math., 80 (1977), 313-331.

[3] T. C. Christofides and E. Vaggelatou, A connection between supermodular ordering and positive/negative association, J. Multivariate. Anal., 88 (2004), 138-151.

[4] H. W. Block, W. S. Griffths and T. H. Savits, L-superadditive structure functions, Adv. Appl. Probab., 21 (1989), 919-929. 
[5] N. Eghbal, M. Amini and A. Bozorgnia, Some maximal inequalities for quadratic forms of negative superadditive dependence random variables, Statist. Probab. Lett., 80 (2010), 587591.

[6] N. Eghbal, M. Amini and A. Bozorgnia, On the Kolmogorov inequalities for quadratic forms of dependent uniformly bounded random variables, Statist. Probab. Lett., 81 (2011), 11121120.

[7] Y. Shen, X. J. Wang, W. Z. Yang and S. H. Hu, Almost sure convergence theorem and strong stability for weighted sums of NSD random variables, Acta Math. Sin. (Engl. Ser.), 29 (2013), 743-756.

[8] Y. Shen, X. J. Wang and S. H. Hu, On the strong convergence and some inequalities for negatively superadditive dependent sequences, J. Inequal. Appl., 2013 (2013), 448.

[9] X. J. Wang, X. Deng, L. L. Zheng and S. H. Hu, Complete convergence for arrays of rowwise negatively superadditive dependent random variables and its applications, Statistics, 48(4) (2014), 834-850.

[10] X. H. Wang, X. Q. Li and S. H. Hu, On the complete convergence of weighted sums for an array of rowwise negatively superadditive dependent random variables, Science Asia, 42(1) (2016), 66-74.

[11] M. O. Cebrera, A. Rosalsky and A. Volodin, Some theorems on conditional mean convergence and conditional almost sure convergence for randomly weighted sums of dependent random variables, TEST, 21 (2012), 369-385.

[12] W. Z. Yang, Y. W. Wang, X. H. Wang and S. H. Hu, Complete moment convergence for randomly weighted sums of martingale differences, J. Inequal. Appl., 2013 (2013), 396.

[13] X. Q. Li, Z. R. Zhao, W. Z. Yang and S. H. Hu, The inequalities of randomly weighted sums of pairewise NQD sequences and its applications to limit theory, J. Math. Inequal., 11 (2017), 323-334.

[14] X. J. Wang, T. C. Hu, A. Volodin and S. H. Hu, Complete convergence for weighted sums and arrays of rowwise extended negatively dependent random variables, Comm. Stat. Theor. Meth., 42(13) (2013), 2391-2401.

[15] A. Adler and A. Rosalsky, Some general strong laws for weighted sums of stochastically dominated random variables, Stoch. Anal. Appl., 5 (1987), 1-16.

[16] S. H. Sung, Moment inequalities and complete moment convergence for arrays of dependent random variables, J. Inequal. Appl., 2009 (2009), Article ID 271265.

[17] Q. Y. Wu, Probability Limit Theory for Mixed Sequence, China Science Press, 2006. 